\title{
Structure and Hydration of Asymmetric Polyelectrolyte Multilayers as Studied by Neutron Reflectometry: Connecting Multilayer Structure to Superior Membrane Performance
}

\author{
Isaac J. Gresham," Dennis M. Reurink, Stuart W. Prescott, Andrew R. J. Nelson, Wiebe M. de Vos,
} and Joshua D. Willott*, ${ }^{*}$

Cite This: Macromolecules 2020, 53, 10644-10654

Read Online

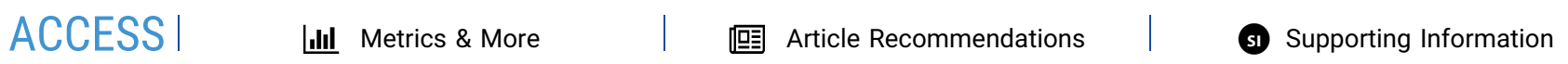

ABSTRACT: Porous membranes coated with so-called asymmetric polyelectrolyte multilayers (PEMs) have recently been shown to outperform commercial membranes for micropollutant removal. They consist of open support layers of poly(styrene sulfonate) (PSS)/poly(allylamine) (PAH) capped by denser and more selective layers of either $\mathrm{PAH} /$ poly(acrylic acid) (PAA) or $\mathrm{PAH} /$ Nafion. Unfortunately, the structure of these asymmetric PEMs, and thus their superior membrane performance, is poorly understood. In this work, neutron reflectometry (NR) is employed to elucidate the multilayered structure and hydration of these asymmetric PEMs. NR reveals that the multilayers are indeed asymmetric in structure, with distinct bottom and top multilayers
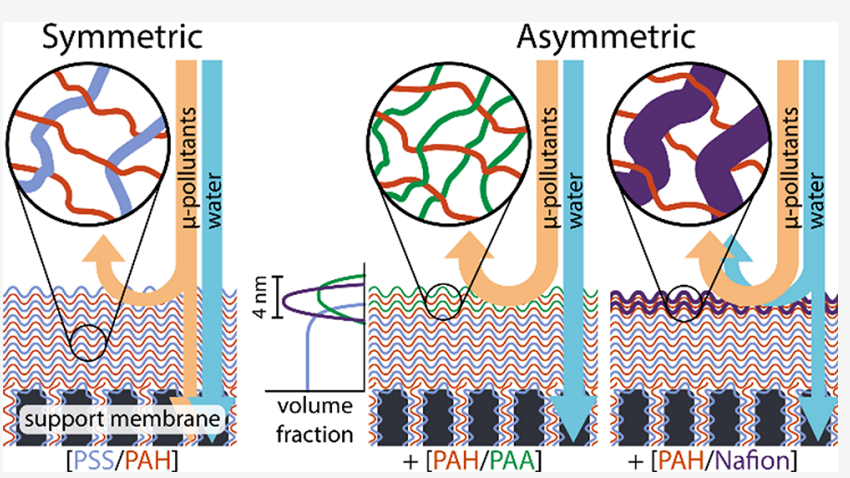
when air-dried and when solvated. The low hydration of the top [PAH/Nafion] multilayer, together with the low water permeance of comparable [PAH/Nafion]-capped PEM membranes, demonstrate that it is a reduction in hydration that makes these separation layers denser and more selective. In contrast, the [PAH/ $\mathrm{PAA}$ ] capping multilayers are more hydrated than the support [PSS/PAH] layers, signifying that, here, densification of the separation layer occurs through a decrease in the mesh size (or effective pore size) of the top layer due to the higher charge density of the PAH/PAA couple compared to the PSS/PAH couple. The [PAH/PAA] and [PAH/Nafion] separation layers are extremely thin $(\sim 4.5$ and $\sim 7 \mathrm{~nm}$, respectively), confirming that these asymmetric PEM membranes have some of the thinnest separation layers ever achieved.

\section{INTRODUCTION}

Layer-by-layer (LBL) deposition of oppositely charged polyelectrolytes (PEs) to form polyelectrolyte multilayers (PEMs) has proven to be a powerful approach to control the physicochemical behavior of interfaces. ${ }^{1,2}$ PEMs are found in a wide variety of applications; they can be used as coatings to modify surface properties in optical, ${ }^{3}$ electrochemical, ${ }^{4}$ flame retardant, ${ }^{5}$ and biomedical ${ }^{6}$ applications. Soon after the publication of the LBL approach for PEM assembly by Decher et al., 7 PEMs were also used in membrane science as they allow for the fabrication of new composite membranes with ultrathin and defect-free separation layers for nanofiltration applications. $^{8-11}$ The internal structure of PEMs is particularly important for membrane applications, with neutron reflectometry a powerful approach to elucidate their structure in situ.

PEMs are particularly versatile coatings as their thickness, density, hydration, and charge can be readily fine-tuned to suit the desired application by careful selection of the PEs and variation of the salt concentration and $\mathrm{pH}$ of the coating solutions. ${ }^{2,12}$ Multilayer hydration is especially important for PEM-coated membranes as higher layer hydration typically increases water permeance. ${ }^{13}$ The choice of PE couple is important for controlling hydration; using weak PEs such as poly(L-glutamic acid) and poly(L-lysine) tends to give more hydrated layers ${ }^{14}$ than those made with strong PEs like poly(styrene sulfonate) (PSS) and poly(diallyldimethylammonium chloride). ${ }^{15}$ The salt concentration of the PE coating solutions, together with salt identity, have also been shown to influence the ultimate hydration of multilayers. ${ }^{15}$ In addition, PEMs with only a few layers tend to be more hydrated than those with many layers. ${ }^{16}$ PEM hydration can also be dependent on whether the multilayer is terminated by the polyanion or polycation, ${ }^{16-18}$ the so-called

Received: August 17, 2020

Revised: November 4, 2020

Published: November 18, 2020

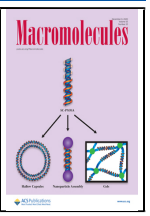


"odd-even" effect. Even the temperature during multilayer preparation affects eventual PEM hydration. ${ }^{19}$

Multilayers containing [PSS/poly(allylamine) (PAH)], $[$ poly(acrylic acid) (PAA)/PAH], and $[\mathrm{Nafion} / \mathrm{PAH}]$ have been shown to be especially relevant for membrane applications. $^{20-22}[\mathrm{PSS} / \mathrm{PAH}]$ multilayers have been widely studied, and their layer-by-layer build-up is linear at low salt concentrations $(<100 \mathrm{mM})$, resulting in thin layers ${ }^{23}$ that are typically non-stoichiometric with an excess of PAH of $\sim 30 \%{ }^{24}$ At higher salt concentrations $(>100 \mathrm{mM})$, the build-up becomes non-linear (exponential), ${ }^{23}$ a phenomenon seen in all PEM systems. ${ }^{12,25}$ [PAA/PAH] multilayers are similarly well-studied, and since both these PEs are weakly charged, the $\mathrm{pH}$ of the PE coating solutions becomes a crucial parameter. PAA is a weak polyacid, and PAH is a weak polybase; their effective $\mathrm{p} K_{\mathrm{a}}$ values (in dilute solution) are $\sim 4.5^{26}$ and $\sim 8.7{ }^{27}$ respectively. At $\mathrm{pH}$ values where both these PEs are highly charged (i.e., $\mathrm{pH} \mathrm{6-7.5),} \mathrm{thinner} \mathrm{multilayers} \mathrm{are} \mathrm{favored,} \mathrm{while}$ at $\mathrm{pH}$ values where only one of the PEs is charged, thicker layers are found. ${ }^{28-31}$ Studies on PEMs containing Nafion, a hydrophobic and highly fluorinated anionic PE, are limited. $22,32,33$ Reurink et al. showed that terminating a [PSS/PAH] multilayer with a final layer of Nafion results in a reduction in multilayer swelling from $\sim 30 \%$ to $\sim 15 \%$. ${ }^{22}$

As previously mentioned, PEMs are closely linked to the field of membrane science. Here, the standard practice is to coat porous support membranes with PEMs in a LBL fashion to create new composite membranes with thin and selective separation layers. Unfortunately, this is not as straightforward as it may first seem. The problem is that, while thin layers are desired, coating a thin layer does not result in complete pore closure of the support membrane, and thus much thicker multilayers are needed to create defect-free membranes. ${ }^{34}$ In addition, the effective thickness of the PEM is even greater since the multilayer also builds inside the pores. Typically, this problem leads to two scenarios: (i) membranes with thick and dense PEM coatings that have high solute retentions but very low water permeances and (ii) membranes with slightly thinner yet more open PEM coatings that have higher water permeances but reduced solute retentions. Recently, te Brinke and co-workers ${ }^{21}$ showed that it is possible to resolve this problem by coating so-called asymmetric PEMs, a concept first invoked by the Bruening group. ${ }^{35,36}$ In this approach, ${ }^{21}$ a relatively thick [PSS/PAH] multilayer was coated first (support layer, closing the pores) followed by a much thinner $[\mathrm{PAA} / \mathrm{PAH}]$ multilayer coated on top (separation layer). The improvement in membrane performance was striking, with on average $98 \%$ micropollutant retention and 5-10 times higher water permeance compared to commercial membranes with similar micropollutant retentions. Similar performance improvement was reported for Nafion-terminated and Nafioncontaining asymmetric PEM-coated membranes. ${ }^{22}$

For asymmetric PEMs topped with $[\mathrm{PAA} / \mathrm{PAH}]$ and $[\mathrm{Nafion} / \mathrm{PAH}]$, the mechanism by which the asymmetric layers improve the separation performance of the membranes is unclear. For example, the asymmetric top layer may lead to densification of the multilayer (reduction in effective mesh/ pore size) by lowering hydration, as postulated for the Nafioncontaining systems, ${ }^{22}$ or the top multilayer may have similar hydration yet a smaller mesh size than the support layer, leading to a separation layer with a lower effective pore size. ${ }^{37}$ Therefore, to understand the excellent performance of both these asymmetric PEM systems, it is crucial to determine their layer structure and hydration. Unfortunately, measuring the solvated thickness and hydration of these thin separation layers is only possible on model substrates. Moreover, for asymmetric PEMs, conventional techniques like ellipsometry and quartz crystal microbalance (QCM) are unable to provide this information as they cannot distinguish between the "bottom" (support) and the "top" (separation) multilayers. Indeed, at this moment, it is not known if these multilayers build asymmetrically or if the bottom and top layers become mixed. Contrary to ellipsometry and QCM, neutron reflectometry (NR) is an ideal tool for studying these asymmetric PEMs, as NR allows for the variation of scattering contrast between the different layers of the system through selective deuteration of the $\operatorname{PE}(\mathrm{s})$ and solvent. ${ }^{38,39}$

NR has been widely used to study the properties of symmetric PEMs. ${ }^{12,40}$ This study is the first to examine the detailed structure of asymmetric PEMs. NR measurements of symmetric multilayers, containing carefully positioned deuterated layers of PE, revealed that PEMs can indeed be highly ordered multilayer films. ${ }^{41,42}$ Moreover, the degree of interpenetration between adjacent PE layers can be controlled by parameters such as the ionic strength ${ }^{43}$ and temperature ${ }^{19,44}$ used during layer preparation. Another strength of using NR is the ability to measure the total (or true) hydration of PEMs. Here, NR is able to quantify both the amount of water that leads to layer swelling and the amount of water present in the space between the PE chains by examining changes in both layer thickness and scattering length density (SLD), respectively. ${ }^{45}$ In this work, four different PEMs are studied: a symmetric $[\mathrm{PSS} / \mathrm{PAH}]$ multilayer and three asymmetric multilayers consisting of support layers of $[\mathrm{PSS} / \mathrm{PAH}]$ and top separation layers of either $[\mathrm{PAA} / \mathrm{PAH}]$ or $[\mathrm{Nafion} / \mathrm{PAH}]$. NR is used to investigate the structure, thickness, and hydration of the PEMs with a particular focus on resolving differences in the support and separation layers of the asymmetric multilayers. The new findings are then related to the performance of analogous asymmetric PEM membranes recently reported by Reurink et $a l^{22}$ and te Brinke et al. ${ }^{21}$

\section{EXPERIMENTAL SECTION}

Materials. Poly(allylamine) (PAH, $\bar{M}_{w}: \sim 150 \mathrm{~kg} \cdot \mathrm{mol}^{-1}$, as a $40 \mathrm{wt}$ $\%$ aqueous solution) was purchased from Nittobo Medical (Japan). Poly(styrene sulfonate) (PSS, $\bar{M}_{w}: \sim 200 \mathrm{~kg} \cdot \mathrm{mol}^{-1}$, as a 30 wt $\%$ aqueous solution), poly(acrylic acid) (PAA, $\bar{M}_{w}: \sim 100 \mathrm{~kg} \cdot \mathrm{mol}^{-1}$, as a 35 wt \% aqueous solution), Nafion (as a $5 \mathrm{wt} \%$ solution in a mixture of lower aliphatic alcohols and $15-20 \%$ water), and branched poly(ethyleneimine) (PEI, $M_{W}: \sim 25 \mathrm{~kg} \cdot \mathrm{mol}^{-1}$, as an entirely PEI solution) were all purchased from Sigma-Aldrich/Merck. Deuterated PAA (d-PAA, $\bar{M}_{w}: \sim 96 \mathrm{~kg} \cdot \mathrm{mol}^{-1}$, as a dry solid) was purchased from Polymer Source (Canada). Sodium chloride (SANAL-P, pharmaceutical quality) was purchased from AkzoNobel. All polyelectrolytes and chemicals were used without further purification (Figure 1). $\mathrm{pH}$ adjustments of the PE solutions were performed using a minimal volume of dilute solutions of sodium hydroxide $(\mathrm{NaOH}$, Alfa Aesar) or hydrochloric acid ( $\mathrm{HCl}$, Sigma-Aldrich/Merck). Milli-Q water $\left(18.2 \mathrm{M} \Omega \cdot \mathrm{cm}\right.$ at $25^{\circ} \mathrm{C}$, Millipore) was used to prepare all dip-coating and rinse solutions. For spectroscopic ellipsometry, silicon wafers with an $\sim 2.5 \mathrm{~nm}$ native $\mathrm{SiO}_{2}$ layer were purchased from Silicon Valley Microelectronics (USA). For specular NR experiments, single side polished silicon wafers of $101.6 \mathrm{~mm}$ diameter and $10 \mathrm{~mm}$ thickness with an $\sim 2.0 \mathrm{~nm}$ native oxide layer were purchased from EL-CAT Inc. (USA).

PEM Preparation. Before PEM preparation, the silicon wafer substrates underwent an $\mathrm{O}_{2}$ plasma treatment for $5 \mathrm{~min}$ to remove any organic contamination before being rinsed with Milli- $\mathrm{Q}$ water and 


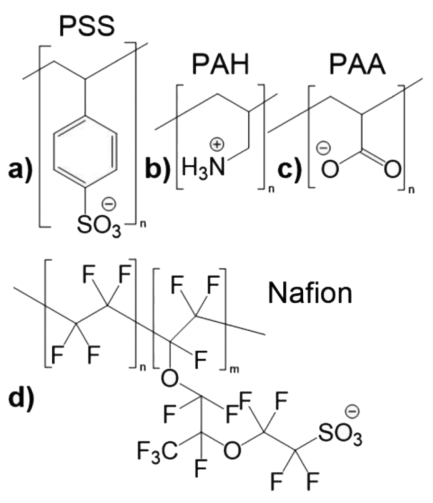

Figure 1. Polyelectrolytes used to prepare the multilayers: (a) poly(styrene sulfonate) (PSS), (b) poly(allylamine) (PAH), (c) poly(acrylic acid), and (d) Nafion.

then dried under a stream of $\mathrm{N}_{2}$. PEM samples for NR were prepared by the dip-coating method, where the silicon wafer substrate is alternately immersed in solutions of polycation and polyanion with rinsing steps in between. In this study, $0.1 \mathrm{~g} / \mathrm{L} \mathrm{PE}$ solutions containing $50 \mathrm{mM} \mathrm{NaCl}$ were used together with $50 \mathrm{mM} \mathrm{NaCl}$ rinsing solutions. All PE solutions were adjusted to a $\mathrm{pH}$ of $6.0 \pm 0.1$. For the Nafion solutions, a 80:20 wt \% ethanol:water solvent mixture was used. All PE adsorption steps were performed for $5 \mathrm{~min}$, and each rinsing was performed thrice for $5 \mathrm{~min}$ each. The LBL assembly of the PEs was repeated until the desired number of layers was obtained, with the polycation/polyanion changed when desired. Following completion of the dip-coating process, all PEMs were soaked in Milli$\mathrm{Q}$ water for $30 \mathrm{~min}$. For all PEMs, the first layer was branched PEI. PEMs with an initial layer of PEI have been shown to be more laterally homogeneous (desirable for NR experiments) as the branched PEI acts as a uniform anchoring network for the consecutive layer formation. ${ }^{46,47}$ All prepared PEMs had 22 individual PE layers, so the final (or terminating) layer was always a polyanion (either PSS, d-PAA, or Nafion). Four different PEMs were prepared by dip-coating and studied by NR. One of the PEMs was the symmetric system $[\mathrm{PEI}]_{1}[\mathrm{PSS} / \mathrm{PAH}]_{10.5}$. The other three PEMs were the asymmetric systems, $[\mathrm{PEI}]_{1}[\mathrm{PSS} / \mathrm{PAH}]_{8.5}[\mathrm{PAH} / \mathrm{d}-\mathrm{PAA}]_{2}, \quad[\mathrm{PEI}]_{1}[\mathrm{PSS} /$ $\mathrm{PAH}]_{8.5}[\mathrm{PAH} / \mathrm{Nafion}]_{2}$, and $[\mathrm{PEI}]_{1}[\mathrm{PSS} / \mathrm{PAH}]_{7.5}[\mathrm{PAH} / \mathrm{d}-\mathrm{PAA}]_{3}$. As all the PEMs have an initial PEI layer, from now on, PEI will be omitted from the naming scheme. For spectroscopic ellipsometry measurements, the PEMs were not prepared by dip-coating, but rather they were prepared in situ, allowing multilayer build-up to be studied as a function of layer number.

Spectroscopic Ellipsometry. Ellipsometry measurements were performed using a J.A. Woollam M-2000 V spectroscopic ellipsometer (USA) with data analysis completed using the J.A. Woollam CompleteEASE software package. The air-dry thickness of the final dip-coated PEM samples was measured at angles of incidence of $65^{\circ}$, $70^{\circ}$, and $75^{\circ}$ across the wavelength of light range of 370-1000 nm for five different surface locations. To calculate the air-dry thickness values, the recorded ellipsometric quantities $\Psi$ and $\Delta$ were fitted using a multiple-layer model consisting of sequentially an infinite $\mathrm{Si}$ layer, a $2.5 \mathrm{~nm} \mathrm{SiO}$ layer, and a polymer layer (of uniform density) representing the entire PEM described by a Cauchy model; $n(\lambda)=A$ $+B / \lambda^{2}$, where $n$ is the refractive index of the layer, $\lambda$ is the wavelength, and $A$ and $B$ are the Cauchy coefficients. For in situ ellipsometry, the polycation and polyanion solutions $(\mathrm{pH} 6,0.1 \mathrm{~g} / \mathrm{L} \mathrm{PE}$ in $50 \mathrm{mM}$ $\mathrm{NaCl}$ ) were alternatingly flowed through the fluid cell with rinse solution $(50 \mathrm{mM} \mathrm{NaCl})$ in between. PEM build-up was followed by recording $\Psi$ and $\Delta$ as a function of time over the spectral range of $370-1000 \mathrm{~nm}$ at a fixed angle of incidence of $75^{\circ}$. The in situ ellipsometric data were fitted using a multiple-layer model consisting of sequentially an infinite $\mathrm{Si}$ layer, a $2.5 \mathrm{~nm} \mathrm{SiO}_{2}$ layer, a linear effective medium approximation (EMA) layer of solvent (water) and polymer of unknown thickness and composition, and an ambient water layer. $\mathrm{For} \mathrm{Si}, \mathrm{SiO}_{2}$, and water, dielectric values available within the software package were used, while the $A$ and $B$ Cauchy coefficients (1.439 and 0.010, respectively) for the PEMs were taken from air-dry measurements of the final films. The thickness of the native $\mathrm{SiO}_{2}$ layer was measured on all unmodified wafer samples (i.e., before PEM coating), and this data was used to constrain the fitting and improve the robustness of the data processing. The asymmetric PEMs could not be modeled as two distinct regions due to low refractive index contrast between the different polyelectrolytes. The fact that these layers are very thin $(<30 \mathrm{~nm})$ is an additional obstacle to implementing models with increased complexity (i.e., models with more fittable parameters) because the change in $\Psi$ as a function of thickness is minimal. ${ }^{48,49}$

Neutron Reflectometry. Specular NR measurements were carried out on the PLATYPUS time-of-flight reflectometer ${ }^{50}$ at the OPAL $20 \mathrm{MW}$ reactor at the Australian Nuclear Science and Technology Organisation, Sydney, Australia. NR was measured as a function of the scattering vector, $Q=(4 \pi / \lambda) \sin \theta$, where $\lambda$ is the wavelength and $\theta$ is the angle of incidence. Measurements of each dipcoated PEM sample were made in air and against three contrasts of $\mathrm{D}_{2} \mathrm{O}, \mathrm{H}_{2} \mathrm{O}$, and $\mathrm{CM}$ (CM refers to a $70: 30 \mathrm{v} / \mathrm{v} \mathrm{H}_{2} \mathrm{O}: \mathrm{D}_{2} \mathrm{O}$ mix intended to match the SLD of the [PSS/PAH] base layer), with a background electrolyte of $5 \mathrm{mM} \mathrm{NaCl}$. Air measurements were carried out in ambient conditions, with a temperature of $21^{\circ} \mathrm{C}$ and an approximate relative humidity of $50 \%$; measurements were made with angles of incidence of $0.65^{\circ}$ and $3.0^{\circ}$, resulting in a usable $Q$ range of $0.008-0.25 \AA^{-1}$. After characterization in air, the samples were placed in solid-liquid cells, and the cells were filled with $\mathrm{D}_{2} \mathrm{O}$. Measurements were performed first in $\mathrm{D}_{2} \mathrm{O}$, then $\mathrm{CM}$, and lastly $\mathrm{H}_{2} \mathrm{O}$. Solution exchange was carried out in situ by pumping $20 \mathrm{~mL}$ of the new solution through the cell at a rate of $1 \mathrm{~mL} \cdot \mathrm{min}^{-1}$; the cell volume is $\sim 0.5 \mathrm{~mL}$. Importantly for all cases, the PEM samples were allowed to equilibrate for at least $2 \mathrm{~h}$ in the given solvent environment before being measured; time-resolved NR measurements support that the films were equilibrated, see Figure S1. For the solvated symmetric $[\mathrm{PSS} / \mathrm{PAH}]_{10.5}$ system, three angles of incidence $\left(0.8^{\circ}, 2.0^{\circ}\right.$, and $\left.5.0^{\circ}\right)$ were measured, resulting in a $Q$ range of $0.01-0.4 \AA^{-1}$. Analysis during data collection revealed that not all three angles were required; $3.8^{\circ}$ (a maximum $Q$ of $0.3 \AA^{-1}$ ) was more than sufficient to reach background. Consequently, data were collected at angles of $0.8^{\circ}$ and $3.8^{\circ}$ for subsequent solid-liquid measurements. An angle of $0.8^{\circ}$ was sufficiently shallow to capture the $\mathrm{Si}: \mathrm{D}_{2} \mathrm{O}$ critical edge, allowing subsequent solvent contrasts to be correctly scaled. Data was reduced using the refnx reflectometry analysis package ${ }^{51}$ using standard procedures, resulting in an overall $Q$ resolution of approximately $5.1 \%$.

NR Data Analysis. Data were analyzed in the ref $n x$ reflectometry package $^{51}$ using a model constructed from a series of layers, each defined by a thickness, roughness, and SLD value as well as an optional solvent volume fraction. The layer roughness parameter describes the width of the Gaussian error function that blends consecutive layers. The effect of roughness on the calculated reflectometry profile is calculated using the Nevot-Croce approximation. The Nevot-Croce approximation works by multiplying the reflectance coefficient of an interface by an exponential damping term and does not propagate to reflectance coefficients of other interfaces. The reflectivity of micro-sliced SLD profiles, (with zero roughness between 1 and $2 \AA$ layers), Figure S2, compares well to the reflectivity calculated by the Nevot-Croce approach. The layer roughness parameter is used to describe both physical roughnesses (i.e., the roughness of the $\mathrm{SiO}_{2}$ surface) and regions of intermixing between consecutive layers (i.e., the intermixing between the support and separation PEMs). Each layer represented a component of the physical interface, with each of the native $\mathrm{SiO}_{2}$ layer, PEI base layer, $[\mathrm{PSS} / \mathrm{PAH}]$ layer, and asymmetric capping layer of [PAH/PAA] or $[\mathrm{PAH} /$ Nafion] being represented by a single slab. As in previous work on PEM systems, it was not necessary to model the individual polycation/polyanion layers separately; rather, a single layer was assigned to the distinct deuterated top layer and undeuterated bottom layer sections. ${ }^{17}$ This model was refined against all four NR datasets (air, $\mathrm{D}_{2} \mathrm{O}, \mathrm{CM}$, and $\mathrm{H}_{2} \mathrm{O}$ ) simultaneously. The SLD of $\mathrm{D}_{2} \mathrm{O}$ was allowed to vary between 6 and 6.37 to account for contamination with 
$\mathrm{H}_{2} \mathrm{O}$, while the SLDs of $\mathrm{CM}$ and $\mathrm{H}_{2} \mathrm{O}$ were taken as 1.5 and -0.54 $\AA^{-2}$, respectively. The addition of the $5 \mathrm{mM} \mathrm{NaCl}$ did not significantly change the SLD of the water. The thickness, solvent fraction, SLD, and roughness of the $\mathrm{SiO}_{2}$ layer were allowed to vary within sensible bounds and were common to all contrasts, while the thickness and roughness of corresponding polymer slabs was shared across the three water contrasts. Constraining the thicknesses in this manner circumvents finding identical symmetric solutions (caused by the phase problem) during optimization and allows for the calculation of a compositional depth profile. ${ }^{52}$ The SLD values of the polymer slabs were unique for each contrast. While it would have been possible for the polymer layers to share a common SLD and solvent fraction value, this would have assumed that the SLD of the polymer layers was independent of the isotope of the solvating water. Our analysis later shows that this is indeed the case, but we do not make that assumption here.

The model was optimized against the four datasets using the differential evolution algorithm of Storn and Price, ${ }^{53}$ as implemented in SciPy. ${ }^{54}$ The optimizer was run 10 times, with the profile with the lowest $\chi^{2}$ term being selected as the profile of best fit. During this optimization, parameters were allowed to vary within sensible bounds. Most notably, the SLD of the polymer layers in each solvent environment could vary to account for solvation by the different water isotopes. After optimization, the posterior probability distributions of the parameters were determined by a parallel-tempered Markov chain Monte Carlo (MCMC) method, as implemented by Vousden et al. in ptemcee. 55 For details regarding the implementation of MCMC in $r e f n x$, the reader is referred to the work of Nelson and Prescott. ${ }^{51}$ To facilitate reproducibility, all data has been made available online alongside a template of the analysis method. ${ }^{56}$

Quantifying Total PEM Hydration. In studying the solvation behavior of PEMs, it is important to calculate the degree of hydration correctly by partitioning hydrating water into two classes: swelling water and so-called void water. ${ }^{45}$ The swelling water volume fraction $\left(\phi_{\text {swell }}\right)$ is the value typically reported for PEM hydration and is calculated directly from the change in thickness upon solvation:

$$
\phi_{\text {swell }}=\frac{d_{\text {solvated }}-d_{\text {dry }}}{d_{\text {solvated }}}
$$

where $d_{\text {dry }}$ is the dry thickness and $d_{\text {solvated }}$ is the solvated thickness. However, there always exists a discrepancy between the hydration values calculated from the thickness $\left(\phi_{\text {swell }}\right)$ and the hydration calculated from the change in the SLD. Delajon et al. ${ }^{18}$ and others ${ }^{15,45}$ posit that the reason for this disagreement is the presence of voids/ space between the PE chains in the dry polymer matrix. In a dehydrated state ( $0 \%$ relative humidity), these voids are filled with air, but when exposed to a solvent, they fill with water $\left(\phi_{\text {void }}\right)$. This event does not change the PEM thickness, but it does change its SLD. The total amount of solvent in the film is then given by

$$
\phi_{\text {total }}=\phi_{\text {swell }}+\phi_{\text {void }}
$$

It must be stressed that the void volume is filled with water upon film solvation; at this point, the distinction between $\phi_{\text {swell }}$ and $\phi_{\text {void }}$ is notional. Although we did not measure our dry films at $0 \%$ relative humidity (the relative humidity of the atmosphere was approximately $50 \%)$, we still adopt the $\phi_{\text {void }}$ notation to refer to hydration that results in a change in SLD but not a change in thickness. It is worth noting that it is the void volume (not the void fraction) that stays constant as the layer swells, such that

$$
\phi_{\text {void }}=\phi_{\text {dry void }}\left(1-\phi_{\text {swell }}\right)
$$

Dodoo et al. ${ }^{15}$ derived a formula for the polymer fraction of the dry layer $\left(1-\phi_{\text {dry void }}\right)$, and hence $\phi_{\text {void }}$ (through eq 3$)$ by assuming that the SLD of the dry voids is zero, that the molecular volume of the PEs in question is independent of the solvation state and that there are no labile protons within the PEM. This method has been used to provide self-consistent approximations for the total hydration of PEMs using dry measurements (at $0 \%$ relative humidity) and a single solvent contrast. $^{45,57}$ Here, we instead utilize multiple water contrasts to calculate the effective solvated SLD of the layers:

$$
\rho_{\text {PE layer }}=\frac{\rho_{\text {layer }, 1} \rho_{\text {water }, 2}-\rho_{\text {layer }, 2} \rho_{\text {water }, 1}}{\left(\rho_{\text {layer }, 1}-\rho_{\text {water }, 1}\right)-\left(\rho_{\text {layer }, 2}-\rho_{\text {water }, 2}\right)}
$$

where $\rho_{\mathrm{PE}}$ layer is the composition-weighted average of the polyelectrolyte SLD values (excluding solvent), and $\rho_{\text {layer, } i}$ and $\rho_{\text {water, } i}$ are the SLD of the layer and water in water contrast $i$, respectively. See the Supporting Information for the full derivation. This approach makes some of the same assumptions as Dodoo et al., ${ }^{15}$ namely, that there are no labile protons in the system. However, by measuring with three water contrasts, $\rho_{\text {PElayer }}$ can be calculated for each of the three solvent pairs. Then, if the SLD values calculated from the different contrast pairs (i.e., $\mathrm{D}_{2} \mathrm{O}-\mathrm{H}_{2} \mathrm{O}, \mathrm{D}_{2} \mathrm{O}-\mathrm{CM}$, and $\mathrm{CM}-\mathrm{H}_{2} \mathrm{O}$ ) are selfconsistent, the effect of labile protons and other imperfections can be neglected. We calculate $\rho_{\text {PElayer }}$ by averaging the values from all available contrast pairs and report the standard deviation as a measure of self-consistency. This PE layer SLD can then be used to calculate $\phi_{\text {total }}$ :

$$
\phi_{\text {total }}=\frac{\rho_{\text {solvated }}-\rho_{\mathrm{PE} \mathrm{layer}}}{\rho_{\text {solvent }}-\rho_{\mathrm{PE} \mathrm{layer}}}
$$

Again, this process was repeated for all measured contrasts, and the results averaged. Once $\phi_{\text {swell }}$ and $\phi_{\text {total }}$ are known, finding $\phi_{\text {void }}$ is then trivial. Consistency was then checked by calculating dry void fraction through Equation 3 and comparing it with:

$$
\phi_{\text {dry void }}=\frac{\rho_{\text {dry }}-\rho_{\text {PE layer }}}{\rho_{\text {dry void }}-\rho_{\text {PE layer }}}
$$

where $\rho_{\text {dry }}$ is the SLD of the dry PE layer, and $\rho_{\text {dryvoid }}$ is between 0 (air) and $-0.56 \times 10^{-6} \AA^{-2}\left(\mathrm{H}_{2} \mathrm{O}\right)$ as we did not measure the dry film thickness under a vacuum. The dry void fractions calculated through both methods were typically within 5\% of one another (see Table S1), indicating that our method is consistent with the approach of Dodoo et al. ${ }^{15} \mathrm{~A}$ worked example detailing our approach is supplied in the Supporting Information.

\section{RESULTS AND DISCUSSION}

This section is divided into three main parts. First, the layerby-layer build-up of the symmetric and asymmetric PEMs, as measured by ellipsometry, is presented and discussed. Next, NR studies of the structure and hydration of both the symmetric and asymmetric multilayers are given, and lastly, the insights gained from NR are linked to the performance of comparable asymmetric PEM-coated membranes.

Layer-by-Layer Build-Up. Figure 2 presents the LBL build-up as measured by in situ ellipsometry for the four different PEMs studied in this work. The build-up is shown from the 7.5 bilayer onward in order to highlight the differences in the solvated thickness of the PEMs when the final asymmetric layers are added (full multilayer build-up is presented in Figure S3). All multilayers consist of a total of 10.5 bilayers, are polyanion terminated, and are prepared from $0.1 \mathrm{~g} / \mathrm{L} \mathrm{PE}$ coating solutions ( $50 \mathrm{mM} \mathrm{NaCl}$ adjusted to $\mathrm{pH}$ 6). As expected for the chosen coating conditions, the LBL buildup of the symmetric $[\mathrm{PSS} / \mathrm{PAH}]_{10.5}$ multilayer is linear, with the thickness of each bilayer roughly the same. ${ }^{23}$ For the asymmetric PAA-containing systems, after the linear build-up of the bottom $[\mathrm{PSS} / \mathrm{PAH}]$ layers, the final $[\mathrm{PAA} / \mathrm{PAH}]$ layers build in a non-linear or exponential fashion. ${ }^{30,31}$ The nonlinear build-up of the PAA/PAH layers is more apparent for the $[\mathrm{PSS} / \mathrm{PAH}]_{7.5}[\mathrm{PAH} / \mathrm{d}-\mathrm{PAA}]_{3}$ multilayer than the $[\mathrm{PSS} /$ $\mathrm{PAH}]_{8.5}[\mathrm{PAH} / \mathrm{d}-\mathrm{PAA}]_{2}$ multilayer, and this results in an overall thicker, more solvated layer. In comparison, the 


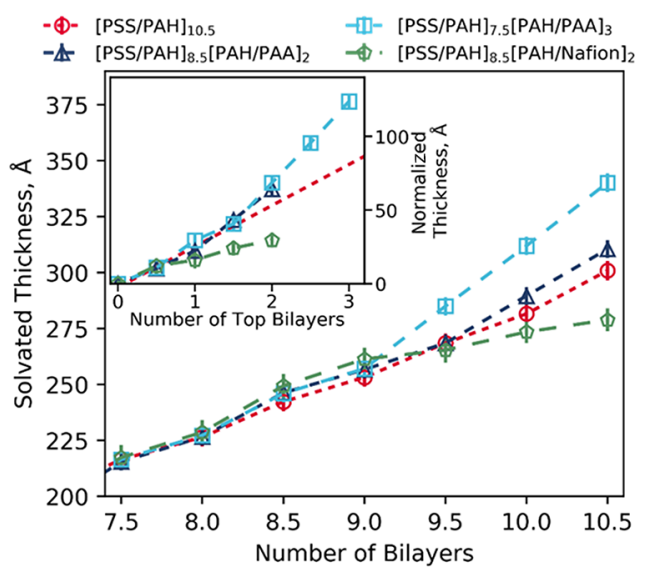

Figure 2. Solvated thickness as a function of bilayer number for the symmetric and asymmetric PEMs as measured by ellipsometry (for complete data sets from 0 to 10.5 bilayers, see Figure S3). The inset plot shows the solvated thickness of the asymmetric top layer as a function of bilayers added to allow for comparison of the top layer build-up.

$[\mathrm{PSS} / \mathrm{PAH}]_{8.5}[\mathrm{PAH} / \mathrm{Nafion}]_{2}$ multilayer has a much lower solvated thickness.

Study of PEM Structure. NR is sensitive to the SLD of interfacial components in the direction normal to the interface. SLD is analogous to an optical refractive index and is dependent on the density and the nuclei present in a material. Here, the top PE layer has a higher SLD than the bottom layer (through its partial deuteration or use of the perfluorinated Nafion), which allows the layered structure of the PEMs to be investigated with NR.

The structure of the four PEM multilayers was determined via co-refinement of multiple NR profiles collected from systems with different solvent contrasts using a single structural model. The optimized models of the PEMs were in good agreement with all collected datasets. SLD profiles and corresponding reflectometry profiles are presented in full in Figure 3. To begin, we discuss the SLD profiles for the [PSS/ $\mathrm{PAH}]_{10.5}$ multilayer across the four different contrasts (Figure 3a). On the left of the SLD profiles is the Si block followed by the native $\mathrm{SiO}_{2}$ layer and the thin base layer of branched PEI, which is used to allow for the formation of smoother multilayers. $^{46,47}$ After the PEI layer comes the [PSS/PAH] multilayer of uniform density followed by the surrounding medium, which is either air, $\mathrm{D}_{2} \mathrm{O}$, the $\mathrm{CM}$ solution, or $\mathrm{H}_{2} \mathrm{O}$.

For the asymmetric PEMs, it is immediately apparent from the SLD profiles (Figure 3c,e,g) that the bottom (support) and top (separation) multilayers are distinct from one another for both the air-dry and solvated measurements. The films referred to as "air-dry" are measured against air and will have a degree of residual water present in them, either from deposition/ rinsing or from humidity in the atmosphere (our analysis accounts for this). A single uniform layer was sufficient to model the $[\mathrm{PSS} / \mathrm{PAH}]_{10.5}$ dataset, while two layers were required for all asymmetric datasets. Attempts to model all datasets with a single uniform layer were unsuccessful for all but the symmetric $[\mathrm{PSS} / \mathrm{PAH}]_{10.5}$ dataset (see Figure S4). The intermixing between bottom and top multilayers was represented in our structural model using a roughness term. In the case of the $[\mathrm{PAH} / \mathrm{d}-\mathrm{PAA}]$ structures, the degree of interlayer mixing is high, resulting in the apparent layer SLD (from the SLD profiles) not matching the value of the SLD modeling parameter; the reported SLD for the top layer is then taken from the SLD profile (Figure 3). Models with a high degree of interlayer mixing were validated by a micro-slicing approach (see Figure S3). The reported SLD value is sampled from each profile at the location of the SLD-maximum in the $\mathrm{H}_{2} \mathrm{O}$ contrasts. The $\mathrm{H}_{2} \mathrm{O}$ contrast is used to find the "center" of the layer because the low solvent SLD results in the top layer forming a clear SLD peak (Figure 3).

Another prominent feature seen in the SLD profiles is the change in layer SLD when the isotope of the solvating water is varied, which is most pronounced for the $[\mathrm{PAH} / \mathrm{d}-\mathrm{PAA}]$ layers and least pronounced for the $[\mathrm{PAH} / \mathrm{Nafion}]$ layers. These changes indicate that a significant portion of the volume of the $[\mathrm{PSS} / \mathrm{PAH}]$ and $[\mathrm{PAH} / \mathrm{d}-\mathrm{PAA}]$ layers is made up of water, while very little water is present in the $[\mathrm{Nafion} / \mathrm{PAH}]$ layer; the water content of these layers is analyzed and discussed in detail in the following sections.

From the three solvent contrasts, the approximate SLD values for each of the PEMs can be determined via eq 4; these values are presented in Table 1 . Two main inferences can be drawn from the calculated SLD values. First, the SLD of the $[\mathrm{PAH} / \mathrm{d}-\mathrm{PAA}]$ layer is lower for the $[\mathrm{PSS} / \mathrm{PAH}]_{8.5}[\mathrm{PAH} / \mathrm{d}$ $\mathrm{PAA}]_{2}$ sample than the $[\mathrm{PSS} / \mathrm{PAH}]_{7.5}[\mathrm{PAH} / \mathrm{d}-\mathrm{PAA}]_{3}$ sample, which indicates that the intermixing region between the two polymer layers is greater than the thickness of the two [PAH/ d-PAA] layers. Second, the high SLD of the undeuterated $\left[\mathrm{PAH} /\right.$ Nafion] layer $\left(3.64 \pm 0.04 \times 10^{-6} \AA^{-2}\right)$ suggests that the layer contains little $\mathrm{PAH}$; the SLD of pure Nafion is estimated to be $4.1 \times 10^{-6} \AA^{-2}$ (based on molar volume calculations using approaches described by van Krevelen and Nijenhuis ${ }^{60}$ ), which is in line with the reported SLD of thin films of Nafion $\left(4.4 \times 10^{-6} \AA^{-2}\right) .^{61}$

Determining PEM Hydration. The layer SLD values presented in Table 1 and the SLD profiles in Figure 3 allow for layer thicknesses as well as $\phi_{\text {swell }}, \phi_{\text {void }}$, and $\phi_{\text {total }}$ (eqs 1,3 , and 5 , respectively) to be calculated for all four PEMs. These data are presented in Table 2, alongside values from ellipsometry measurements. Void fraction values have been omitted for clarity but may be easily calculated as the difference of $\phi_{\text {swell }}$ and $\phi_{\text {total }}$ (eq 2). As expected from the ellipsometry data (Figure 2 and Table 2 ), the $[\mathrm{PSS} / \mathrm{PAH}]_{7.5}[\mathrm{PAH} / \mathrm{d}-\mathrm{PAA}]_{3}$ multilayer is slightly thicker than the $[\mathrm{PSS} / \mathrm{PAH}]_{8.5}[\mathrm{PAH} / \mathrm{d}-$ $\mathrm{PAA}]_{2}$ multilayer with the NR data revealing a significant difference between the solvated thickness of the top [PAH/dPAA] layers for these systems, $70 \AA$ vs $44 \AA$, respectively. A description of how the separation layer thicknesses are calculated can be found in the Supporting Information, Section S7. For comparable asymmetric multilayers, te Brinke et al. roughly estimated the thicknesses of the top [PAH/PAA] layers to be $\sim 160 \AA$ for the three-layer system and $\sim 40 \AA$ for the two-layer system. ${ }^{21}$ The discrepancy between the ellipsometry measurements of te Brinke et al. and the thicknesses measured here by NR could be due to penetration of $[\mathrm{PAH} / \mathrm{PAA}]$ into the support multilayer of [PSS/PAH]. While these estimates based on ellipsometry measurements are reasonable, $\mathrm{NR}$ reveals that the $[\mathrm{PAH} / \mathrm{d}-\mathrm{PAA}]_{3}$ separation layer is thinner than first reported. The PEMs studied here are very thin $(20-40 \mathrm{~nm})$, and this is around the limit of what can be reasonably expected for absolute thickness measurements from ellipsometry. ${ }^{48}$ Conversely, this thickness range is ideal for neutron reflectometry studies. The reflectometry data possess Kiessig fringes whose spacing is inversely proportional to layer thickness, allowing thickness changes to be probed at 

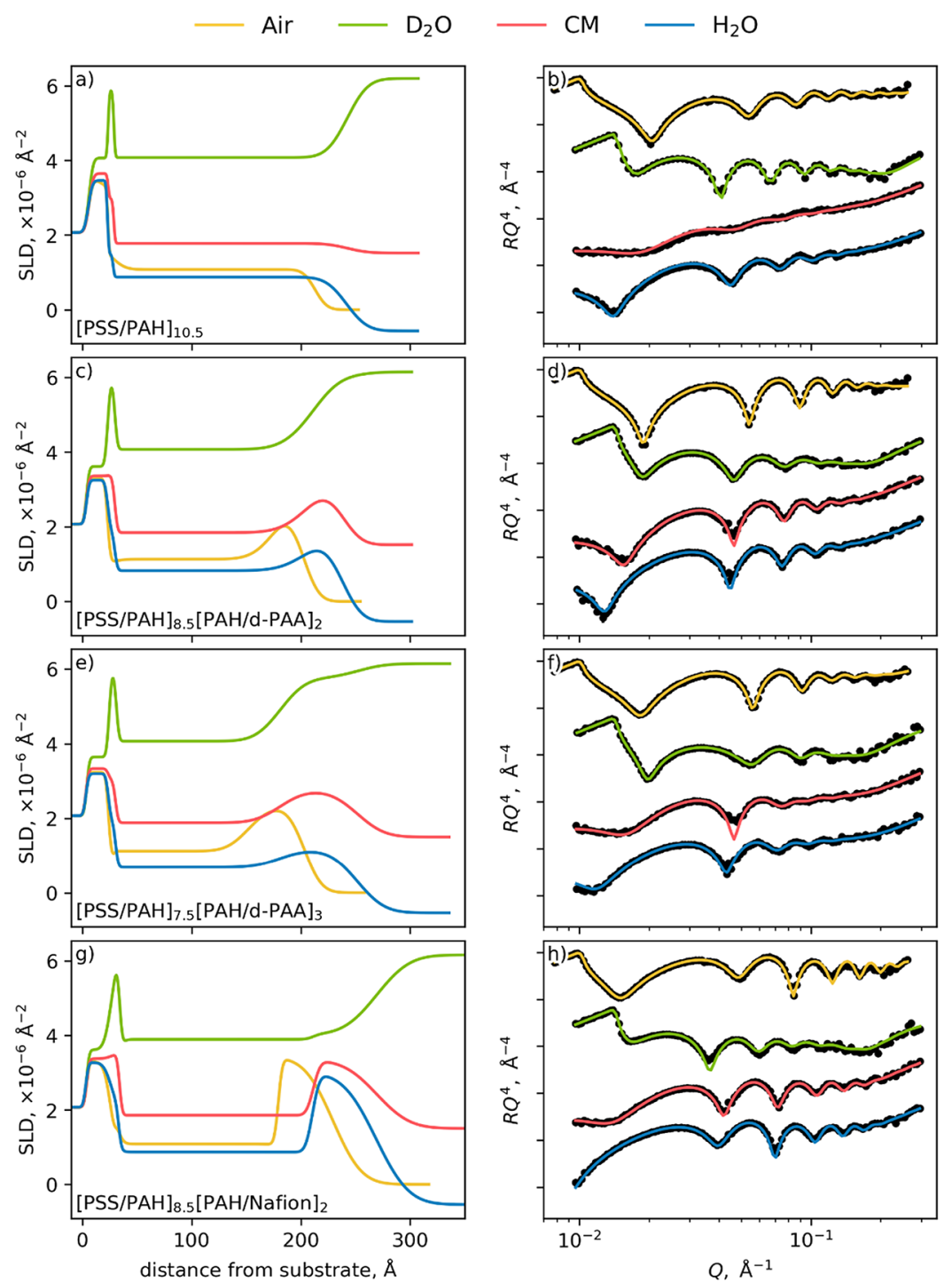

Figure 3. SLD profiles (left panels) of best fit with corresponding reflectometry profiles (right panels) collected from the four different PEMs. Each PEM was studied in four contrasts, $\mathrm{D}_{2} \mathrm{O}$, a $70: 30 \mathrm{H}_{2} \mathrm{O}: \mathrm{D}_{2} \mathrm{O}(\mathrm{v} / \mathrm{v})$ contrast match $(\mathrm{CM})$ mix, $\mathrm{H}_{2} \mathrm{O}$, and air. $\mathrm{SiO}_{2}$ was the backing medium for the air measurements. For the asymmetric PEMs, the top (separation) multilayer was distinct from the bottom (support) multilayer. Thickness, swelling, and hydration of the layers are quantified in Table 2 . The reflectometry profiles are vertically offset for clarity. The high SLD value $(\sim 6 \times$ $10^{-6} \AA^{-2}$ ) of the PEI layer in the $\mathrm{D}_{2} \mathrm{O}$ contrast was required to fit the high-Q features of the reflectometry profiles (fringe at $\left.Q \approx 0.2\right)$ and is consistent across all the PEMs studied in this work; similar SLD values for PEI in $\mathrm{D}_{2} \mathrm{O}$ have been reported in the literature. ${ }^{58,59}$

Table 1. Empirically Derived Layer SLD Values from eq 4

\begin{tabular}{|c|c|}
\hline & $\begin{array}{l}\text { empirical SLD values of the PE layers } \\
\qquad\left(\times 10^{-6} \AA^{-2}\right)^{a}\end{array}$ \\
\hline$[\mathrm{PSS} / \mathrm{PAH}]_{10.5}$ & $2.05 \pm 0.06$ \\
\hline \multicolumn{2}{|l|}{$[\mathrm{PSS} / \mathrm{PAH}]_{8.5}[\mathrm{PAH} / \mathrm{d}-\mathrm{PAA}]_{2}$} \\
\hline Bottom PEM (PSS/PAH) & $2.11 \pm 0.07$ \\
\hline Top PEM (PAH/d-PAA) & $3.94 \pm 0.13$ \\
\hline \multicolumn{2}{|l|}{$[\mathrm{PSS} / \mathrm{PAH}]_{7.5}[\mathrm{PAH} / \mathrm{d}-\mathrm{PAA}]_{3}$} \\
\hline Bottom PEM (PSS/PAH) & $2.16 \pm 0.26$ \\
\hline Top PEM (PAH/d-PAA) & $4.66 \pm 0.15$ \\
\hline \multicolumn{2}{|l|}{$[\mathrm{PSS} / \mathrm{PAH}]_{8.5}[\mathrm{PAH} / \mathrm{Nafion}]_{2}$} \\
\hline Bottom PEM (PSS/PAH) & $2.07 \pm 0.10$ \\
\hline Top PEM (PAH/Nafion) & $3.64 \pm 0.04$ \\
\hline \multicolumn{2}{|c|}{$\begin{array}{l}{ }^{a} \text { Average of the values calculated for the } \mathrm{D}_{2} \mathrm{O}-\mathrm{H}_{2} \mathrm{O}, \mathrm{D}_{2} \mathrm{O}-\mathrm{CM} \text {, and } \\
\mathrm{CM}-\mathrm{H}_{2} \mathrm{O} \text { contrast pairs with uncertainties being the standard } \\
\text { deviation of the values. The reported SLD value is sampled from } \\
\text { each profile at the location of the SLD-maximum in the } \mathrm{H}_{2} \mathrm{O} \text { contrast. }\end{array}$} \\
\hline
\end{tabular}

the Angstrom level. Our analysis predominantly relies on neutron reflectometry for absolute thickness and internal structure measurements. However, in situ preparation of samples for NR experiments would not have been feasible due to the scarcity of allocated NR beamtime. In contrast, ellipsometry is able to determine the relative thicknesses of films and changes in the PEM thickness during an in situ layerby-layer deposition.

Both NR and ellipsometry reveal that $\phi_{\text {swell }}$ (solvent uptake due to change in thickness) is greater for the [PAH/d-PAA] separation layers compared to the $[\mathrm{PSS} / \mathrm{PAH}]$ support layers, while it is much lower for the [PAH/Nafion] layer. The PEI thicknesses are not included in the thickness of either the airdry or solvated [PSS/PAH] base layer; however, PEI layer thickness did vary during fitting (see Table S3). Our analysis was unable to distinguish between the air-dry PEI layer and the [PSS/PAH] layer as the PEI layer is very thin and appeared to have an SLD value similar to that of the [PSS/PAH] layer; this 
Table 2. Thickness, Swelling, and Hydration of the PEMs Studied in This Work

\begin{tabular}{|c|c|c|c|c|c|c|c|}
\hline & \multicolumn{3}{|c|}{ ellipsometry } & \multicolumn{4}{|c|}{ neutron reflectometry } \\
\hline & \multicolumn{2}{|c|}{ thickness $(\AA)^{a}$} & \multirow{2}{*}{$\frac{\text { hydration (\%) }}{\phi_{\text {swell }}{ }^{\mathrm{c}}}$} & \multicolumn{2}{|c|}{${\text { thickness }(\AA)^{b}}^{b}$} & \multicolumn{2}{|c|}{ hydration (\%) } \\
\hline & air-dry & solvated & & air-dry & solvated & $\phi_{\text {swell }}^{c}$ & $\phi_{\text {total }}^{\mathrm{d}}$ \\
\hline$[\mathrm{PSS} / \mathrm{PAH}]_{10.5}$ & $232 \pm 5$ & $301 \pm 4$ & $23 \pm 2$ & $187 \pm 0$ & $214 \pm 0$ & $12.7 \pm 0.0$ & $46.9 \pm 3.0$ \\
\hline$[\mathrm{PSS} / \mathrm{PAH}]_{8.5}[\mathrm{PAH} / \mathrm{d}-\mathrm{PAA}]_{2}$ & $243 \pm 3$ & $310 \pm 4$ & $22 \pm 2$ & $179 \pm 1^{f}$ & $212 \pm 0^{f}$ & $15.8 \pm 0.4$ & $47.2 \pm 1.8^{\mathrm{e}}$ \\
\hline Bottom PEM (PSS/PAH) & & & & $143 \pm 1^{f}$ & $168 \pm 0^{f}$ & $15.0 \pm 0.4$ & $46.3 \pm 1.9$ \\
\hline Top PEM (PAH/PAA) & & & & $36 \pm 0^{f}$ & $44 \pm 0^{f}$ & $18.9 \pm 1.0$ & $54.8 \pm 3.8$ \\
\hline$[\mathrm{PSS} / \mathrm{PAH}]_{7.5}[\mathrm{PAH} / \mathrm{d}-\mathrm{PAA}]_{3}$ & $251 \pm 4$ & $340 \pm 4$ & $26 \pm 1$ & $174 \pm 2$ & $220 \pm 1$ & $21.4 \pm 0.2$ & $52.6 \pm 6.9^{\mathrm{e}}$ \\
\hline Bottom PEM (PSS/PAH) & & & & $123 \pm 2$ & $150 \pm 0$ & $18.6 \pm 0.4$ & $47.2 \pm 10$ \\
\hline Top PEM (PAH/PAA) & & & & $51 \pm 1$ & $70 \pm 1$ & $28.1 \pm 0.5$ & $64.1 \pm 4.0$ \\
\hline$[\mathrm{PSS} / \mathrm{PAH}]_{8.5}[\mathrm{PAH} / \text { Nafion }]_{2}$ & $263 \pm 6$ & $279 \pm 5$ & $6 \pm 3$ & $199 \pm 0$ & $235 \pm 0$ & $15.4 \pm 0.0$ & $35.5 \pm 3.7^{\mathrm{e}}$ \\
\hline Bottom PEM (PSS/PAH) & & & & $149 \pm 0$ & $177 \pm 0$ & $16.0 \pm 0.0$ & $42.4 \pm 4.9$ \\
\hline Top PEM (PAH/Nafion) & & & & $50 \pm 0$ & $58 \pm 0$ & $13.8 \pm 0.1$ & $14.4 \pm 1.4$ \\
\hline
\end{tabular}

${ }^{a}$ Data for corresponding PEMs prepared on smaller silicon wafers suitable for ellipsometry. All reported thicknesses do not include the PEI layer. Uncertainties are the standard deviation from at least three distinct measurements taken at different locations on the sample. ${ }^{b}$ Uncertainties are taken from the posterior probability distribution for the parameter. ${ }^{c}$ Calculated using eq $1 .{ }^{d}$ Calculated from eq 5 and SLDs in Table $1 .{ }^{e}$ Thickness weighted average of bottom and top layer values. ${ }^{f}$ Due to large interlayer mixing to thickness ratios, layer thickness was calculated via the method described in Section S8.

contributes to the minor discrepancies in $\phi_{\text {swell }}$ between the [PAH/PSS $]$ base layers across the four PEMs seen in the NR results. Discrepancies may also arise from differences in the experimental setup, sample, and measurement technique. Ellipsometry was used to study the build-up of the PEMs in situ, while NR was applied to the complete PEMs. In addition, hysteric effects associated with air-drying and re-solvating of the films may result in small changes in the measured thickness values.

The total hydration, $\phi_{\text {total }}$, for the [PSS/PAH] layer is similar across all NR datasets. Consistent with ellipsometry, the overall hydration of the $[\mathrm{PSS} / \mathrm{PAH}]_{7.5}[\mathrm{PAH} / \mathrm{d}-\mathrm{PAA}]_{3}$ multilayer is greater than the $[\mathrm{PSS} / \mathrm{PAH}]_{8.5}[\mathrm{PAH} / \mathrm{d}-\mathrm{PAA}]_{2}$ multilayer; NR reveals that this is a consequence of $\phi_{\text {total }}$ being larger for the separation layer in $[\mathrm{PSS} / \mathrm{PAH}]_{7.5}[\mathrm{PAH} / \mathrm{d}-\mathrm{PAA}]_{3}$ compared to $[\mathrm{PSS} / \mathrm{PAH}]_{8.5}[\mathrm{PAH} / \mathrm{d}-\mathrm{PAA}]_{2}$. Furthermore, $[\mathrm{PAH} / \mathrm{d}-\mathrm{PAA}]$ is more hydrated than [PSS/PAH]. In comparison to the bottom layer of $[\mathrm{PSS} / \mathrm{PAH}]$, the $[\mathrm{PAH} / \mathrm{d}-\mathrm{PAA}]$ layers are more hydrated, while the [PAH/Nafion] layer is much less hydrated. The low hydration of the [PAH/Nafion] layer is in line with the water content reported for thin films of Nafion studied by NR. ${ }^{61,62}$ In Table $2, \phi_{\text {total }}$ is determined from the (calculated) SLD of the polyelectrolyte pair and the (measured) SLD of the layer. The calculation of $\phi_{\text {total }}$ requires very few assumptions (see Section S1 of the Supporting Information) and is in line with reported solvent fractions for PEMs. ${ }^{15,18,45} \phi_{\text {swell }}$ (solvent uptake due to change in thickness) is calculated from the change in thickness between the air-dry state $(50 \%$ ambient humidity) and the solvated state. Our choice of dry state (ambient, air-dry) may differ slightly from other works, which measure the films under a vacuum or a dry nitrogen stream. From the work that examines the swelling of polyelectrolytes as a function of relative humidity ${ }^{16}$ and Flory-Huggins theory, ${ }^{63}$ we predict that our air-dry films (50\% relative humidity) will be no more than $10 \%$ thicker than chemically comparable truedry films. In this current study, we are primarily concerned with the hydrated structure of asymmetric PEMs and the relative hydration of these layers. As such, we present $\phi_{\text {swell }}$ values for comparison between datasets.

The values in Table 1 and SLD profiles in Figure 3 can be used to produce approximate volume fraction profiles of the PEMs, as shown in Figure 4. These profiles possess the same

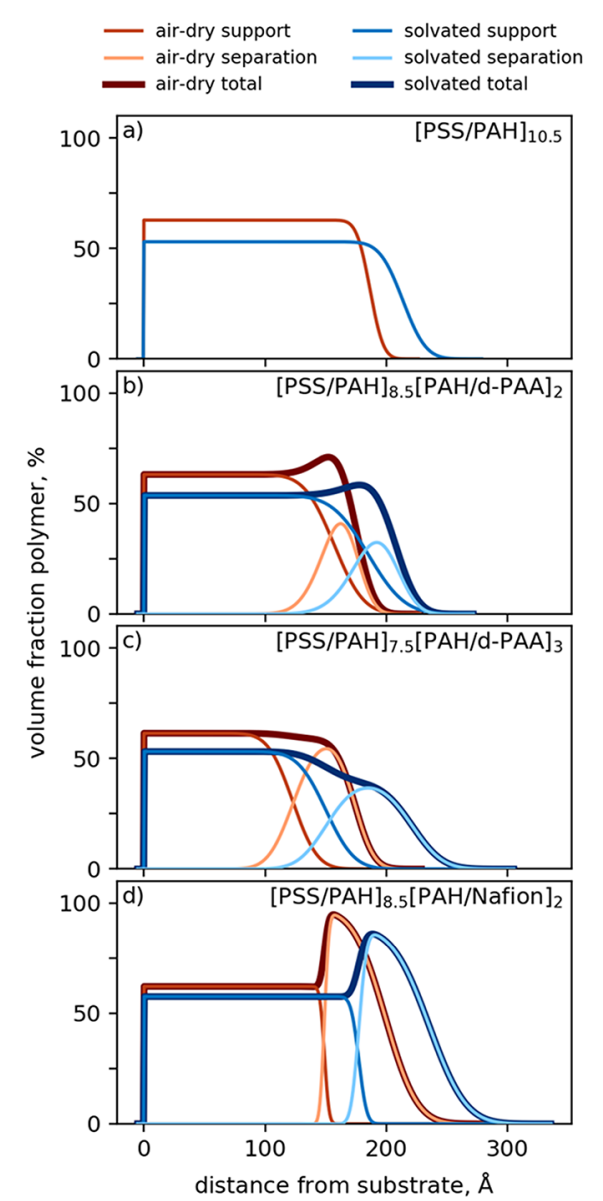

Figure 4. Solvated and air-dry polymer volume fraction profiles extracted from SLD profiles for all studied multilayers. The volume fraction profiles emphasize the structural difference between the $[\mathrm{PAH} / \mathrm{d}-\mathrm{PAA}](\mathrm{b}, \mathrm{c})$ and $[\mathrm{PAH} / \mathrm{Nafion}](\mathrm{d})$ top separation layers. Nafion (d) is distinct from the base layer, while d-PAA exhibits significant intermixing with the base layer. For the PAA-containing samples, the intermixing region is greater than two deposited [PAH/ d-PAA] bilayers (b) but smaller than three (c). The uncertainties in the polymer volume fractions are the same as the total solvent fraction uncertainties of Table 2 . 
Table 3. Performance of Similar Symmetric and Asymmetric PEM Membranes

\begin{tabular}{|c|c|c|c|c|}
\hline & $\begin{array}{l}\text { water permeance } \\
\left(\mathrm{L} \cdot \mathrm{m}^{-2} \cdot \mathrm{h}^{-1} \cdot \mathrm{bar}^{-1}\right)\end{array}$ & $\begin{array}{l}\text { water permeance decrease due to } \\
\text { asymmetric layers }\end{array}$ & $\begin{array}{l}\mathrm{MWCO}^{\mathrm{b}} \\
\left(\mathrm{g} \cdot \mathrm{mol}^{-1}\right)\end{array}$ & $\begin{array}{l}\text { average micropollutant } \\
\text { retention (\%) }\end{array}$ \\
\hline \multicolumn{5}{|l|}{ Data from te Brinke et al. } \\
\hline$[\mathrm{PSS} / \mathrm{PAH}]_{10.5}$ & $15.2 \pm 0.6$ & & $321 \pm 3$ & $93.2 \pm 0.3$ \\
\hline$[\mathrm{PSS} / \mathrm{PAH}]_{8.5}[\mathrm{PAH} / \mathrm{PAA}]_{2}$ & $12.8 \pm 0.6$ & $\sim 16 \%$ & $265 \pm 2$ & $97.7 \pm 0.1$ \\
\hline$[\mathrm{PSS} / \mathrm{PAH}]_{7.5}[\mathrm{PAH} / \mathrm{PAA}]_{3}$ & $8.9 \pm 0.7$ & $\sim 41 \%$ & $246 \pm 5$ & $98.2 \pm 0.5$ \\
\hline \multicolumn{5}{|l|}{ Data from Reurink et al. } \\
\hline$[\mathrm{PSS} / \mathrm{PAH}]_{8.5}$ & $6.2 \pm 0.4$ & & $301 \pm 5$ & $90.0 \pm 0.3$ \\
\hline$[\mathrm{PSS} / \mathrm{PAH}]_{7.5}[\mathrm{PAH} / \mathrm{Nafion}]_{1}$ & $4.8 \pm 0.7$ & $\sim 23 \%$ & $287 \pm 8$ & $92.7 \pm 1.0$ \\
\hline
\end{tabular}

${ }^{a_{T}}$ The exact water permeance values should not be directly compared as the coating conditions and support membranes onto which the PEMs are added are different. Instead, it is better to compare the percentage decrease in water permeance due to the asymmetric coating given in the adjacent column. ${ }^{b}$ Values obtained from the retention of short-chain polyethylene glycol molecules. A lower MWCO value signifies a lower effective membrane pore size.

structural form as the NR models in Figure 3, that is, a series of slabs of a given thickness and SLD joined by a Gaussian roughness of a given breadth (representing interlayer mixing). The volume fraction of each of these layers was calculated from the SLD values of the multilayers (Table 1), the SLD of the layer in $\mathrm{H}_{2} \mathrm{O}$ (Figure 3), and the SLD of $\mathrm{H}_{2} \mathrm{O}$ (taken as -0.56 $\left.\times 10^{-6} \AA^{-2}\right)$. It should be noted that the volume fraction profiles of Figure 4 are approximations, with the uncertainty the same as the uncertainty for the total solvent fractions given in Table 2. Furthermore, the PEM structure is inferred from the SLD profiles, where Gaussian interlayer transitions are assumed, and the polycation and polyanion of a specific multilayer are grouped together. The exact structure of the $[\mathrm{PSS} / \mathrm{PAH}]_{8.5}[\mathrm{PAH} / \mathrm{d}-\mathrm{PAA}]_{2}$ layer was more challenging to resolve than the other PEMs as here the interlayer mixing region between the support and separation layers is comparable to the thickness of the separation layer. This large transition region is consistent between both $[\mathrm{PAH} / \mathrm{d}$ PAA] asymmetric multilayers.

Figure 4 demonstrates that the asymmetry of PEMs is retained upon solvation and that the structures of the Nafioncontaining and PAA-containing separation layers are very different. [PAH/Nafion] forms a layer that is quite distinct from the base multilayer, while $[\mathrm{PAH} / \mathrm{PAA}]$ forms a layer that is more solvated than the base PEM. These differences can be used to explain the performance of these multilayer systems in membrane separation, as discussed below.

Connecting the NR Results with Similar PEM Membranes. In recent studies, ${ }^{21,22}$ asymmetric PAA- and Nafion-containing PEM membranes have been shown to outperform symmetric PEM membranes and even commercial membranes in nanofiltration applications. Table 3 summarizes the key performance parameters for these asymmetric PEM membranes together with the analogous symmetric systems. Both these asymmetric systems have improved separation performances (higher micropollutant retentions and lower molecular weight cut-off (MWCO) values), but they differ in terms of the loss in water permeance associated with the improved selectivity. Specifically, a single layer of [PAH/ Nafion] results in a much greater decrease in water permeance than two layers of $[\mathrm{PAH} / \mathrm{PAA}]$ (compare $\sim 23 \%$ with $\sim 16 \%$ ), suggesting that these membranes function differently. In this section, the NR results are used to clarify the mechanism through which these PEM membranes function.

The first key discovery from $\mathrm{NR}$ is that the $[\mathrm{PAH} / \mathrm{d}-\mathrm{PAA}]$ and $[\mathrm{PAH} / \mathrm{Nafion}]$ top separation layers are distinct from the [PSS/PAH] base layers (see Figures 3 and 4 ). That is, the asymmetry imparted during fabrication is retained in the final hydrated film, although there is some intermixing between the support and separation multilayers. Moreover, NR confirms that the top separation multilayer is very thin, showing that it is indeed possible to create extremely thin membrane separation layers using asymmetric PEMs. The third significant finding is that the hydration $\left(\phi_{\text {total }}\right)$ of the $[\mathrm{PAH} / \mathrm{d}-\mathrm{PAA}]$ and $[\mathrm{PAH} /$ Nafion] layers are markedly different to each other, their respective base layers, and the symmetric [PSS/PAH] multilayer (see Table 2).

Considering the asymmetric Nafion system first, we ask the question: how does this asymmetric multilayer membrane separate molecules? Table 3 shows that the Nafion-terminated PEM membrane has a lower MWCO (i.e., lower effective pore size), a higher average micropollutant retention, and a lower water permeance compared to the symmetric PEM membrane. These three data imply that a denser (lower effective pore size) multilayer was created. Based on the hydrophobicity of the highly fluorinated Nafion PE, Reurink et al. hypothesized that this behavior was due to a reduction in the swelling of the entire PEM when Nafion was introduced; ${ }^{22}$ the hypothesis is supported by ellipsometry thickness measurements that showed swelling was 10-15\% lower for Nafion-terminated multilayers. Our NR results on a comparable Nafioncontaining PEM show that the $[\mathrm{PAH} / \mathrm{Nafion}]$ layer has very low hydration $(\sim 14 \%$ water, see Table 2$)$. Similarly, low hydrations have been reported in NR studies of thin films of Nafion. ${ }^{61,62} \mathrm{NR}$ also reveals that, for the Nafion case, the hydration of $[\mathrm{PSS} / \mathrm{PAH}]$ base layer is similar to that of the symmetric $[\mathrm{PSS} / \mathrm{PAH}]$ multilayer and the base $[\mathrm{PSS} / \mathrm{PAH}]$ layers of the PAA-containing multilayers. Thus, in contrast to Reurink et al.'s hypothesis, it is not the hydration of the entire PEM that decreases but rather only the top layers of [PAH/ Nafion]. Coupling this decrease in top (separation) layer hydration with the significant drop in water permeance of the Nafion-terminated PEM membrane reveals that, here, the most likely separation mechanism is the densification of the multilayer through a reduction in hydration of the [PAH/ Nafion] top multilayer.

Next, we consider the asymmetric PAA-containing systems and ask the same question: how do these asymmetric multilayer membranes separate molecules? Table 3 shows that the PAA-containing PEM membranes have the same performance traits as the Nafion-containing PEM membranes, i.e., lower MWCO values, average higher micropollutant retentions, and lower water permeances compared to the symmetric PEM membrane. However, there is one key 
difference. The reduction in water permeance due to the asymmetric PAA layers is less than for the Nafion layer. Overall, as with the Nafion case, the data of Table 3 implies that a denser separation layer was obtained when the PEM was capped with layers of $[\mathrm{PAH} / \mathrm{PAA}]$. This data alone could naturally lead one to the conclusion that, once again, a denser layer is achieved through a reduction in multilayer hydration. However, the NR results reveal that this is not the case; indeed, the $[\mathrm{PAH} / \mathrm{PAA}]$ layers are more hydrated than the [PSS/PAH] layers. Therefore, the question remains: how does this membrane improve separation now that densification via a reduction in multilayer hydration cannot be the mechanism.

To answer this question, the chemical structures of PEs used and the charge density of the resultant multilayers, defined as the number of ionic groups per number of carbon atoms in the repeat unit of the PE complex/multilayer, should be considered. A PEM can be imagined as a network of ionically cross-linked chains with Ångström-sized pores, ${ }^{9,37}$ in which cross-link density and hence mesh size of the network is governed by the charge density of the PE complex. From the definition above, and neglecting interdigitation of chains from adjacent layers and variations in weak PE ionization, the charge density of a $[\mathrm{PSS} / \mathrm{PAH}]$ multilayer is 0.09 , while for a $[\mathrm{PAH} /$ $\mathrm{PAA}$ ] multilayer, it is 0.17 . According to the structural model of PEMs, one would expect that membranes prepared from [PAH/PAA] will consist of small yet hydrophilic pores, whereas [PSS/PAH] would lead to membranes with larger, more hydrophobic pores. Indeed, this behavior is perfectly reflected in the results of te Brinke et al. ${ }^{21}$ shown in Table 3, where the asymmetric $[\mathrm{PSS} / \mathrm{PAH}]_{8.5}[\mathrm{PAH} / \mathrm{PAA}]_{2}$ and $[\mathrm{PSS} /$ $\mathrm{PAH}]_{7.5}[\mathrm{PAH} / \mathrm{PAA}]_{3}$ membranes have higher micropollutant retentions (and lower MWCO values) and thus lower effective pore sizes than the symmetric $[\mathrm{PSS} / \mathrm{PAH}]_{10.5}$ membranes. In addition, the NR results reveal that $[\mathrm{PAH} / \mathrm{PAA}]$ layers are more hydrated than the [PSS/PAH] layers (compare 55-63\% water content with $\sim 47 \%$ ) supporting the hypothesis that the higher charge density [PAH/PAA] layers are more hydrophilic than the $[\mathrm{PSS} / \mathrm{PAH}]$ layers. Work by Krasemann et al. ${ }^{37}$ on water-ethanol pervaporation using a broad family of PEM membranes of varying charge density ( 0.06 up to 0.25 from the definition above) was the first to show that this structural model for PEM membranes holds. Differences in the amount of intermixing between the top and bottom layers as highlighted in Figures 3 and 4 explains the slightly higher selectivity of the three-layer [PAH/PAA] system over the twolayer $[\mathrm{PAH} / \mathrm{PAA}]$ system, with the thinner two-layer system more affected by interpenetration with the base [PSS/PAH] layer than the thicker three-layer system.

\section{CONCLUSIONS}

We have determined the mechanism by which asymmetric $[\mathrm{PSS} / \mathrm{PAH}][\mathrm{PAH} / \mathrm{PAA}]$ and $[\mathrm{PSS} / \mathrm{PAH}][\mathrm{PAH} / \mathrm{Nafion}]$ polyelectrolyte multilayer membranes achieve promising micropollutant retentions by resolving their structure using NR. These measurements of the in situ structure are supported with ellipsometry measurements of the layer-by-layer build-up of the films. NR reveals that the top multilayer is structurally distinct from the bottom multilayer in both air-dry and solvated conditions, confirming that these asymmetric layers act as distinct separation regions rather than merely modifying the bulk properties of the base layer. These top separation layers are exceptionally thin; two bilayers of [PAH/PAA] were measured to be $\sim 4.5 \mathrm{~nm}$-thick, while the equivalent [PAH/
Nafion] multilayer was $\sim 7 \mathrm{~nm}$-thick. NR reveals that [PAH/ $\mathrm{PAA}$ ] is more hydrated (63\% water by volume) than the underlying [PSS/PAH] layer (47\%), while [PAH/Nafion] is significantly less hydrated (14\%). When taken together with water permeance and MWCO values from the literature, this difference in hydration points to distinct separation mechanisms between the two top separation layers. We hypothesize that asymmetric multilayers of [PSS/PAH] capped with $[\mathrm{PAH} / \mathrm{PAA}]$ provide better micropollutant retention than symmetric [PSS/PAH] multilayers due to their smaller network mesh size, while the asymmetric [PAH/Nafion] topped multilayers improve retention by being physically denser.

\section{ASSOCIATED CONTENT}

\section{Supporting Information}

The Supporting Information is available free of charge at https://pubs.acs.org/doi/10.1021/acs.macromol.0c01909.

Derivation of eq 4 for PE layer SLD, dry void fraction calculations using eqs 3 and 6, example hydration calculation, time-resolved NR data, confirmation of reflectometry calculations, demonstration of singlelayer models for asymmetric PEMs, LBL build-up of PEMs studied by in situ ellipsometry, how separation layer thicknesses are calculated, and lastly the results of MCMC analysis (PDF)

\section{AUTHOR INFORMATION}

\section{Corresponding Author}

Joshua D. Willott - Membrane Science and Technology, Mesa + Institute for Nanotechnology, University of Twente, Enschede 7500, AE, The Netherlands; O orcid.org/00000003-1870-755X; Phone: +31 53 4893405;

Email: j.d.willott@utwente.nl

\section{Authors}

Isaac J. Gresham - School of Chemical Engineering, University of New South Wales, Sydney, NSW 2052, Australia; (i) orcid.org/0000-0002-5648-3756

Dennis M. Reurink - Membrane Science and Technology, Mesa+ Institute for Nanotechnology, University of Twente, Enschede 7500, AE, The Netherlands

Stuart W. Prescott - School of Chemical Engineering, University of New South Wales, Sydney, NSW 2052,

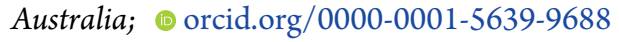

Andrew R. J. Nelson - Australian Nuclear Science and Technology Organisation (ANSTO), Lucas Heights, NSW 2234, Australia; (1) orcid.org/0000-0002-4548-3558

Wiebe M. de Vos - Membrane Science and Technology, Mesa + Institute for Nanotechnology, University of Twente, Enschede 7500, AE, The Netherlands; orcid.org/00000002-0133-1931

Complete contact information is available at: https://pubs.acs.org/10.1021/acs.macromol.0c01909

\section{Author Contributions}

"I.J.G. and J.D.W. contributed equally. The manuscript was written through the contributions of all authors. All authors have approved the final version of the manuscript.

\section{Notes}

The authors declare no competing financial interest. 
The reduced neutron reflectometry data files, as well as a template of the analysis methodology used, are published on Zenodo at DOI: 10.5281 /zenodo.3984647.

\section{ACKNOWLEDGMENTS}

This work was supported by an Australian Centre for Neutron Scattering beamtime grant at ANSTO (P7856). J.D.W. and W.M.d.V. acknowledge funding support from the "Vernieuwingsimpuls" program through project number VIDI 723.015.003 (financed by the Netherlands Organisation for Scientific Research, NWO). I.J.G. thanks the Australian Government and AINSE Ltd. for providing financial assistance (Research Training Program Scholarship and PGRA Award, respectively).

\section{REFERENCES}

(1) Decher, G.; Schlenoff, J. Multilayer Thin Films: Sequential Assembly of Nanocomposite Materials. 2nd ed.; Wiley-VCH: Weinheim, Germany, 2012, DOI: 10.1002/9783527646746.

(2) Borges, J.; Mano, J. F. Molecular Interactions Driving the Layerby-Layer Assembly of Multilayers. Chem. Rev. 2014, 114, 8883-8942.

(3) Hiller, J.; Mendelsohn, J. D.; Rubner, M. F. Reversibly Erasable Nanoporous Anti-Reflection Coatings from Polyelectrolyte Multilayers. Nat. Mater. 2002, 1, 59-63.

(4) Rydzek, G.; Ji, Q.; Li, M.; Schaaf, P.; Hill, J. P.; Boulmedais, F.; Ariga, K. Electrochemical Nanoarchitectonics and Layer-by-Layer Assembly: from Basics to Future. Nano Today 2015, 10, 138-167.

(5) Holder, K. M.; Smith, R. J.; Grunlan, J. C. A Review of Flame Retardant Nanocoatings Prepared using Layer-by-Layer Assembly of Polyelectrolytes. J. Mater. Sci. 2017, 52, 12923-12959.

(6) Boudou, T.; Crouzier, T.; Ren, K.; Blin, G.; Picart, C. Multiple Functionalities of Polyelectrolyte Multilayer Films: New Biomedical Applications. Adv. Mater. 2010, 22, 441-467.

(7) Decher, G.; Hong, J. D.; Schmitt, J. Buildup of Ultrathin Multilayer Films by a Self-Assembly Process: III. Consecutively Alternating Adsorption of Anionic and Cationic Polyelectrolytes on Charged Surfaces. Thin Solid Films 1992, 210-211, 831-835.

(8) Harris, J. J.; Stair, J. L.; Bruening, M. L. Layered Polyelectrolyte Films as Selective, Ultrathin Barriers for Anion Transport. Chem. Mater. 2000, 12, 1941-1946.

(9) Tieke, B.; van Ackern, F.; Krasemann, L.; Toutianoush, A. Ultrathin Self-Assembled Polyelectrolyte Multilayer Membranes. Eur. Phys. J. E: Soft Matter Biol. Phys. 2001, 5, 29-39.

(10) Malaisamy, R.; Bruening, M. L. High-Flux Nanofiltration Membranes Prepared by Adsorption of Multilayer Polyelectrolyte Membranes on Polymeric Supports. Langmuir 2005, 21, 1058710592.

(11) de Grooth, J.; Reurink, D. M.; Ploegmakers, J.; de Vos, W. M.; Nijmeijer, K. Charged Micropollutant Removal With Hollow Fiber Nanofiltration Membranes Based On Polycation/Polyzwitterion/ Polyanion Multilayers. ACS Appl. Mater. Interfaces 2014, 6, 1700917017.

(12) Klitzing, R. V. Internal Structure of Polyelectrolyte Multilayer Assemblies. Phys. Chem. Chem. Phys. 2006, 8, 5012-5033.

(13) Miller, M. D.; Bruening, M. L. Correlation of the Swelling and Permeability of Polyelectrolyte Multilayer Films. Chem. Mater. 2005, 17, 5375-5381.

(14) Halthur, T. J.; Elofsson, U. M. Multilayers of Charged Polypeptides As Studied by in Situ Ellipsometry and Quartz Crystal Microbalance with Dissipation. Langmuir 2004, 20, 1739-1745.

(15) Dodoo, S.; Steitz, R.; Laschewsky, A.; von Klitzing, R. Effect of Ionic Strength and Type of Ions on the Structure of Water Swollen Polyelectrolyte Multilayers. Phys. Chem. Chem. Phys. 2011, 13, 10318-10325.

(16) Wong, J. E.; Rehfeldt, F.; Hänni, P.; Tanaka, M.; Klitzing, R. v. Swelling Behavior of Polyelectrolyte Multilayers in Saturated Water Vapor. Macromolecules 2004, 37, 7285-7289.
(17) de Vos, W. M.; Mears, L. L. E.; Richardson, R. M.; Cosgrove, T.; Barker, R.; Prescott, S. W. Nonuniform Hydration and Odd-Even Effects in Polyelectrolyte Multilayers under a Confining Pressure. Macromolecules 2013, 46, 1027-1034.

(18) Delajon, C.; Gutberlet, T.; Möhwald, H.; Krastev, R. Absorption of Light and Heavy Water Vapours in Polyelectrolyte Multilayer Films. Colloids Surf., B 2009, 74, 462-467.

(19) Gopinadhan, M.; Ivanova, O.; Ahrens, H.; Günther, J.-U.; Steitz, R; Helm, C. A. The Influence of Secondary Interactions during the Formation of Polyelectrolyte Multilayers: Layer Thickness, Bound Water and Layer Interpenetration. J. Phys. Chem. B 2007, 111, 84268434.

(20) Bruening, M. L.; Dotzauer, D. M.; Jain, P.; Ouyang, L.; Baker, G. L. Creation of Functional Membranes Using Polyelectrolyte Multilayers and Polymer Brushes. Langmuir 2008, 24, 7663-7673.

(21) te Brinke, E.; Reurink, D. M.; Achterhuis, I.; de Grooth, J.; de Vos, W. M. Asymmetric Polyelectrolyte Multilayer Membranes with Ultrathin Separation Layers for Highly Efficient Micropollutant Removal. Appl. Mater. Today 2020, 18, 100471.

(22) Reurink, D. M.; te Brinke, E.; Achterhuis, I.; Roesink, H. D. W.; de Vos, W. M. Nafion-Based Low-Hydration Polyelectrolyte Multilayer Membranes for Enhanced Water Purification. ACS Appl. Polym. Mater. 2019, 1, 2543-2551.

(23) Ruths, J.; Essler, F.; Decher, G.; Riegler, H. Polyelectrolytes I: Polyanion/Polycation Multilayers at the Air/Monolayer/Water Interface as Elements for Quantitative Polymer Adsorption Studies and Preparation of Hetero-superlattices on Solid Surfaces. Langmuir 2000, 16, 8871-8878.

(24) Riegler, H.; Essler, F. Polyelectrolytes. 2. Intrinsic or Extrinsic Charge Compensation? Quantitative Charge Analysis of PAH/PSS Multilayers. Langmuir 2002, 18, 6694-6698.

(25) Volodkin, D.; von Klitzing, R. Competing Mechanisms in Polyelectrolyte Multilayer Formation and Swelling: PolycationPolyanion Pairing vs. Polyelectrolyte-Ion Pairing. Curr. Opin. Colloid Interface Sci. 2014, 19, 25-31.

(26) Swift, T.; Swanson, L.; Geoghegan, M.; Rimmer, S. The pHResponsive Behaviour of Poly(acrylic acid) in Aqueous Solution is Dependent on Molar Mass. Soft Matter 2016, 12, 2542-2549.

(27) Fang, M.; Kim, C. H.; Saupe, G. B.; Kim, H.-N.; Waraksa, C. C.; Miwa, T.; Fujishima, A.; Mallouk, T. E. Layer-by-Layer Growth and Condensation Reactions of Niobate and Titanoniobate Thin Films. Chem. Mater. 1999, 11, 1526-1532.

(28) Shiratori, S. S.; Rubner, M. F. pH-Dependent Thickness Behavior of Sequentially Adsorbed Layers of Weak Polyelectrolytes. Macromolecules 2000, 33, 4213-4219.

(29) Choi, J.; Rubner, M. F. Influence of the Degree of Ionization on Weak Polyelectrolyte Multilayer Assembly. Macromolecules 2005, 38, $116-124$.

(30) Bieker, P.; Schönhoff, M. Linear and Exponential Growth Regimes of Multilayers of Weak Polyelectrolytes in Dependence on pH. Macromolecules 2010, 43, 5052-5059.

(31) Vidyasagar, A.; Sung, C.; Losensky, K.; Lutkenhaus, J. L. pHDependent Thermal Transitions in Hydrated Layer-by-Layer Assemblies Containing Weak Polyelectrolytes. Macromolecules 2012, 45, 9169-9176.

(32) Daiko, Y.; Katagiri, K.; Matsuda, A. Proton Conduction in Thickness-Controlled Ultrathin Polycation/Nafion Multilayers Prepared via Layer-by-Layer Assembly. Chem. Mater. 2008, 20, 64056409.

(33) Daiko, Y.; Katagiri, K.; Yazawa, T.; Matsuda, A. Thickness Dependences of Proton Conductivity for Ultrathin Nafion Multilayers prepared via Layer-by-Layer Assembly. Solid State Ionics 2010, 181, 197-200.

(34) de Grooth, J.; Oborný, R.; Potreck, J.; Nijmeijer, K.; de Vos, W. M. The Role of Ionic Strength and Odd-Even Effects on the Properties of Polyelectrolyte Multilayer Nanofiltration Membranes. J. Membr. Sci. 2015, 475, 311-319.

(35) Stair, J. L.; Harris, J. J.; Bruening, M. L. Enhancement of the Ion-Transport Selectivity of Layered Polyelectrolyte Membranes 
through Cross-Linking and Hybridization. Chem. Mater. 2001, 13, 2641-2648.

(36) Liu, X.; Bruening, M. L. Size-Selective Transport of Uncharged Solutes through Multilayer Polyelectrolyte Membranes. Chem. Mater. 2004, 16, 351-357.

(37) Krasemann, L.; Toutianoush, A.; Tieke, B. Self-Assembled Polyelectrolyte Multilayer Membranes with Highly Improved Pervaporation Separation of Ethanol/Water Mixtures. J. Membr. Sci. 2001, 181, 221-228.

(38) Narayanan, T.; Wacklin, H.; Konovalov, O.; Lund, R. Recent Applications of Synchrotron Radiation and Neutrons in the Study of Soft Matter. Crystallogr. Rev. 2017, 23, 160-226.

(39) Delcea, M.; Helm, C. A. X-ray and Neutron Reflectometry of Thin Films at Liquid Interfaces. Langmuir 2019, 35, 8519-8530.

(40) von Klitzing, R.; Köhler, R.; Chenigny, C. Neutron Reflectometry at Polyelectrolyte Multilayers. In Multilayer Thin Films, 2nd ed.; Wiley-VCH: Weinheim, Germany, 2012; pp. 219268.

(41) Schmitt, J.; Gruenewald, T.; Decher, G.; Pershan, P. S.; Kjaer, K.; Loesche, M. Internal Structure of Layer-by-Layer Adsorbed Polyelectrolyte Films: a Neutron and X-ray Reflectivity Study. Macromolecules 1993, 26, 7058-7063.

(42) Korneev, D.; Lvov, Y.; Decher, G.; Schmitt, J.; Yaradaikin, S. Neutron Reflectivity Analysis of Self-Assembled Film Superlattices with Alternate Layers of Deuterated and Hydrogenated Polystyerenesulfonate and Polyallylamine. Phys. B 1995, 213-214, 954-956.

(43) Lösche, M.; Schmitt, J.; Decher, G.; Bouwman, W. G.; Kjaer, K. Detailed Structure of Molecularly Thin Polyelectrolyte Multilayer Films on Solid Substrates as Revealed by Neutron Reflectometry. Macromolecules 1998, 31, 8893-8906.

(44) Gopinadhan, M.; Ahrens, H.; Günther, J.-U.; Steitz, R.; Helm, C. A. Approaching the Precipitation Temperature of the Deposition Solution and the Effects on the Internal Order of Polyelectrolyte Multilayers. Macromolecules 2005, 38, 5228-5235.

(45) Koehler, R.; Steitz, R; von Klitzing, R. About Different Types of Water in Swollen Polyelectrolyte Multilayers. Adv. Colloid Interface Sci. 2014, 207, 325-331.

(46) Kolasińska, M.; Krastev, R.; Warszyński, P. Characteristics of Polyelectrolyte Multilayers: Effect of PEI Anchoring Layer and Posttreatment after Deposition. J. Colloid Interface Sci. 2007, 305, 4656.

(47) Kolasińska, M.; Krastev, R.; Gutberlet, T.; Warszyński, P. Swelling and Water Uptake of PAH/PSS Polyelectrolyte Multilayers. Prog. Colloid Polym. Sci. 2008, 134, 30-38.

(48) Ogieglo, W.; Wormeester, H.; Eichhorn, K.-J.; Wessling, M.; Benes, N. E. In Situ Ellipsometry Studies on Swelling of Thin Polymer Films: A Review. Prog. Polym. Sci. 2015, 42, 42-78.

(49) Tempelman, K.; Kappert, E. J.; Raaijmakers, M. J. T.; Wormeester, H.; Benes, N. E. Dealing with Inaccuracies in the Analysis on Solvent-Induced Swelling of Transparent Thin Films using In Situ Spectroscopic Ellipsometry in the Visible Wavelength Range. Surf. Interface Anal. 2017, 49, 538-547.

(50) James, M.; Nelson, A.; Holt, S. A.; Saerbeck, T.; Hamilton, W. A.; Klose, F. The Multipurpose Time-of-Flight Neutron Reflectometer "Platypus" at Australia's OPAL Reactor. Nucl. Instrum. Methods Phys. Res., Sect. A 2011, 632, 112-123.

(51) Nelson, A. R. J.; Prescott, S. W. refnx: Neutron and X-ray Reflectometry Analysis in Python. J. Appl. Crystallogr. 2019, 52, 193200.

(52) DeCaluwe, S. C.; Kienzle, P. A.; Bhargava, P.; Baker, A. M.; Dura, J. A. Phase Segregation of Sulfonate Groups in Nafion Interface Lamellae, Quantified via Neutron Reflectometry Fitting Techniques for Multi-Layered Structures. Soft Matter 2014, 10, 5763-5776.

(53) Storn, R.; Price, K. Differential Evolution - A Simple and Efficient Heuristic for global Optimization over Continuous Spaces. J. Global Optim. 1997, 11, 341-359.

(54) Virtanen, P.; Gommers, R.; Oliphant, T. E.; Haberland, M.; Reddy, T.; Cournapeau, D.; Burovski, E.; Peterson, P.; Weckesser, W.; Bright, J.; van der Walt, S. J.; Brett, M.; Wilson, J.; Millman, K. J.;
Mayorov, N.; Nelson, A. R. J.; Jones, E.; Kern, R.; Larson, E.; Carey, C. J.; Polat, I.; Feng, Y.; Moore, E. W.; VanderPlas, J.; Laxalde, D.; Perktold, J.; Cimrman, R.; Henriksen, I.; Quintero, E. A.; Harris, C. R.; Archibald, A. M.; Ribeiro, A. H.; Pedregosa, F.; van Mulbregt, P.; SciPy 1.0 Contributors. SciPy 1.0: Fundamental Algorithms for Scientific Computing in Python. Nat. Methods 2020, 17, 261-272.

(55) Vousden, W. D.; Farr, W. M.; Mandel, I. Dynamic Temperature Selection for Parallel Tempering in Markov Chain Monte Carlo Simulations. Mon. Not. R. Astron. Soc. 2015, 455, 1919-1937.

(56) Gresham, I. J.; Reurink, D. M.; Prescott, S. W.; Nelson, A. R. J.; de Vos, W. M.; Willott, J. D. Neutron Reflectometry Datasets for "Structure and Hydration of Asymmetric Polyelectrolyte Multilayers as studied by Neutron Reflectometry: Connecting Multilayer Structure to Superior Membrane Performance". 2020, DOI: 10.5281/zenodo.3984647.

(57) Zerball, M.; Laschewsky, A.; von Klitzing, R. Swelling of Polyelectrolyte Multilayers: The Relation Between, Surface and Bulk Characteristics. J. Phys. Chem. B 2015, 119, 11879-11886.

(58) Wong, J. Y.; Majewski, J.; Seitz, M.; Park, C. K.; Israelachvili, J. N.; Smith, G. S. Polymer-Cushioned Bilayers. I. A Structural Study of Various Preparation Methods Using Neutron Reflectometry. Biophys. J. 1999, 77, 1445-1457.

(59) Félix, O.; Zheng, Z.; Cousin, F.; Decher, G. Are Sprayed LbLfilms Stratified? A First Assessment of the Nanostructure of SprayAssembled Multilayers by Neutron Reflectometry. C. R. Chim. 2009, $12,225-234$.

(60) Van Krevelen, D. W.; Te Nijenhuis, K., Volumetric Properties. In Properties of Polymers, Fourth Edition; Van Krevelen, D. W.; Te Nijenhuis, K., Eds.; Elsevier: Amsterdam, 2009; pp. 71-108.

(61) Kalisvaart, W. P.; Fritzsche, H.; Mérida, W. Water Uptake and Swelling Hysteresis in a Nafion Thin Film Measured with Neutron Reflectometry. Langmuir 2015, 31, 5416-5422.

(62) DeCaluwe, S. C.; Baker, A. M.; Bhargava, P.; Fischer, J. E.; Dura, J. A. Structure-Property Relationships at Nafion Thin-Film Interfaces: Thickness Effects on Hydration and Anisotropic Ion Transport. Nano Energy 2018, 46, 91-100.

(63) Elbs, H.; Krausch, G. Ellipsometric Determination of FloryHuggins Interaction Parameters in Solution. Polymer 2004, 45, 79357942 . 